\title{
Parental Knowledge, Attitudes and Perceptions towards Adolescent Sexual Reproductive Health in Bulawayo
}

\author{
M. Svodziwa, F. Kurete, L. Ndlovu \\ Adult and Continuing Education, Bulawayo Polytechnic, Zimbabwe
}

\begin{abstract}
Parents, the most consistent influence in children's lives, are in unique position to influence young people's health and personal development, and their transition to sexual life. Parents are the first socializing agent of the children. The family stills plays a significant role in the lives of young people. Family environment plays a key role in the timing of sexual and reproductive health transitions in adolescents. Ministry of Health and Family Welfare, 2006), evidence about parent-child interaction and communication, particularly with regard to sensitive matters such as the physical changes associated with puberty, sex, pregnancy and sexually transmitted infections/ HIV is sparse. Following interpretivist methods. The research design that was used in this study was descriptive exploratory design that aimed at collecting information from participants on their ideas and opinions relating to sexual reproductive health. The qualitative method sought in-depth understanding of complex psychological issues and are most useful for answering humanistic "why?" and "how?" questions a qualitative approach to research, collected data through focus group discussions and in depth interviews. This study, probed parental perspectives, suggests that parents are indeed concerned about their children's transition into sexual life, but are constrained by traditional norms, lack of information and limited skills from communicating with and providing them the supportive environment needed to make this transition .Parents should start discussing sexuality with their adolescent children early. Sensitization to both parents and adolescents needs to be done so that any of them can initiate the discussion about sexual and reproductive health issues. Training and evaluation about sexuality to parents on how to communicate with their children of the opposite sex.
\end{abstract}

Keywords : parent, adolescent, sexual reproductive health

\section{INTRODUCTION}

A large body research conducted on sexual reproductive health education documents that there are high levels of risky sexual behaviour among youths in sub-Saharan Africa. Most studies which have been conducted on sexual reproductive health education aretheoretical and have focused on individual risk factors. It is important to note that the studies which have been conducted pay little attention to family and community factors that may decrease the rates of risky sexual behavior, referred to as protective factors. Yet, research conducted in Western contexts has showed that families, especially parents, remain a powerful influence in fostering healthy teenage development and preventing negative social ills, (Davis \& Friel, 2001; Miller et al., 2001).

In this approach, two-parent families are considered homogeneous in strengths: cohesion, connectedness, communication, supervision/monitoring. Yet empirical evidence shows that adolescents and young adults from two-parent families also engage in risky sexual behaviour, especially in the context of severe poverty (Meekers \& Calvès, 1997; Kuate Defo, 2004). As parents do not talk to their children about reproductive health issues, they do not want their children to do anything about sexual and they avoid confronting their adolescent on what they are doing concerning sexuality and this is mostly common among African communities. Hence this study, explored the barriers to communication between parents and adolescents concerning sexual and reproductive issues.

\section{LITERATURE REVIEW}

Parents, likely the most consistent influence in children's lives, are in a unique position to influence young people's health and personal development, and their transition to sexual life (World Health Organization, 2007). When the parent-child relationship is hierarchical and authoritarian, it can have an adverse influence both on how adolescents feel about themselves and the choices they make about behaviours that affect their health (Kirby, 2007; Whitaker and Miller, 2000). 
Further, several studies in developing countries that have focused on the nature and influence of parent-child communication on sexual and reproductive health matters, have concluded that parentchild communication on these matters is uncommon (Biddlecom, Awusabo-Asare and Bankole, 2009; Kiragu et al., 2007; Joint United Nations Programme on HIV/AIDS (UNAIDS), 2008; Cui, Li. and Gao, 2001). Where it takes place, moreover, messages provided by parents on sexuality are usually ambiguous (for example, 'do not play with boys'; Luwaga, 2004). In India, notwithstanding the recognition in policies and programmes of the need to actively engage parents in enabling adolescents to make safe and healthy transitions to adulthood (Ministry of Health and Family Welfare, 2006), evidence about parent-child interaction and communication, particularly with regard to sensitive matters such as the physical changes associated with puberty, sex, pregnancy and sexually transmitted infections/ HIV is sparse. Evidence that is currently available comes largely from the perspectives of young people rather than parents themselves and suggests that socialisation, for many, tends to be authoritarian - particularly for young women - and that communication between parents and both sons and daughters tends to be limited, particularly on sensitive personal matters (see for example, Shekhar et al., 2007; Abraham and Kumar, 1999; Mehra, Savithri and Coutinho, 2002; Alexander et al., 2006; International Institute for Population Sciences (IIPS) and Population Council, 2010), and that strong cultural taboos inhibit talking about sex (Lambert and Wood, 2005).

These studies confirm that communication is limited and highlights a range of obstacles that may inhibit parents' ability or skill in communicating with their children on the physical changes associated with puberty. For example, village-level studies have identified parents' own lack of awareness (Soletti et al., 2009; Garda and Alexander, 2009) and their perception that their children were not at risk of HIV as key factors limiting communication (Soletti et al., 2009); other studies have noted parental discomfort and perceptions that informing their children about sexual matters would lead them to engage in sex (Shetty, Kowli and Patil, n.d; Mahajan and Sharma, 2005; Garda and Alexander, 2009).

For the first time, data are available from several states of India that shed light on socialisation and parent-youth interaction patterns from the perspectives of parents of young men and women as well as from young people themselves. These data allow us to explore the interaction between youth and their parents on sensitive sexual and reproductive health matters both from the perspective of youth themselves and of parents of youth. Perspectives of youth have been described in an earlier work and confirm the limited communication on such matters between parents and children (see IIPS and Population Council, 2010).

Parents are the first socialing agent of the children. The family stills plays a significant role in the lives of young people. Family environment plays a key role in the timing of sexual and reproductive health transitions in adolescents, Ngom, Magadi and Owuor (2003)\& Oyefara (2005). Mench,Grant and Blanc (2006), note that there has been a rise in the proportion of first sex that occurs before marriage. Premarital sex is more likely to occur among adolescents than in the past. Parents and caregivers are expected to monitor and help to shape their behaviour.

Communication between parent and child is a crucial dimension of parenting. There is evidence that suggest that parent-child communication about sex related matters is not very common. Most of the times it is fraught with discomfort especially communication with fathers in Sub-Saharan Africa, ( Awusabo-Asare, Bankole \&Kumi-Kyereme 2008). According to Markham, Lormand, Gloppen, Peskin, Flores, Low, House (2010), it is expected that frequent and positive parent-child communication about sexual and reproductive matters will lower the probability of sexual risk taking by promoting more responsible adolescent behaviour.

Living arrangements of children contribute to understanding parental sexuality communication context. Markham et al (2010), note that children living with single parent are more likely to be sexually active than those in two parent households. On the same note, Babalola, Tambashe and Vondrasek (2005), in their study in $\mathrm{C}^{\wedge}$ oted Ivoire found that female adolescents living in the same household as the father, had a delayed sexual debut as compared to the male adolescents. However UNAIDS (2012) notes that there is little evidence that shows a link between family connectedness and risky sexual behaviour among adolescents. In a study in Cameroon, it revealed that higher quality parent-child relationships decreased the risk of premarital intercourse among adolescents. The study also showed that a lower level of parental monitoring is significantly assosciated with an increase in the risk of premarital sex among adolescents. 
World Health Organisation (2007), has describes programs in a review such as Uganda's Straight Talk Campaign and Families matter in Kenya that have demonstrated the willingness of parent to create a supportive environment for young people and workto improve intrafamily communication about sexuality and sexual risk.

In this paper the researchers, investigate the perspectives of parents themselves, the extent and nature of communication with their children on sexual and reproductive health (SRH) matters, and the factors that inhibit parents from discussing these matters with their children. Sexual and reproductive health matters encompassed in this report cover the physical changes associated with puberty, as well as such matters as sex, pregnancy and sexually transmitted infections/HIV.

Through this lack of communication, the parent-child relationship is destroyed and home becomes a strange world to the learner while the school appears to be the other. For this reason, one cannot avoid noting misbehaviour on the part of child as a result of this gap between school and home. Studies indicate that parents find it not only taboo to discuss sex education with their children but they also perceive it as embarrassing. They find it difficult to talk about sex and are unwilling to pursue any sex education for fear of discussing the moral-laden topic (Bundy \& White 1990; Mouldon \& Tuker 1996). Although no mention of race is made by the authors cited above, it is clear from the researchers' knowledge of African culture in general and that of Xhosa speaking Africans in particular that such topics are not discussed and are left to the instincts of the child.

These values are now 70 disappearing as a result of their encounter with various other cultures, namely, western culture which tends to confuse children and lead them to taking irresponsible sex related decisions. A lack of cross-cultural understanding among Black people creates a serious problem in that it allows no room for observing other cultures. This isolates these communities from other cultures and further alienates black parents from their children who seem to enjoy more exposure to a few other cultures including the western one. Parents in black communities according to Hubley (1990) and Evian (1991), have conservative attitudes and cultural inhibitions that hinder meaningful communication with their children. If true, the foregoing argument explains quite vividly that the gap stated above will continue to widen, unless some form of a cross-cultural exposure is provided and the communities concerned are afforded an opportunity to talk about such topics as sex before marriage, its merits and demerits; self image related talks, their value and so on.

Such talks would help ward off the fear of sexually transmitted diseases, for it has been noted that adolescents have the highest risk of becoming infected, because they are quite skeptical about listening to or accepting information related to sex. It has also been found that young people (adolescents) are less likely to use condoms (Gohel et al 1997 \& Nkosi 1999). This could only be attributed to the fact that parents are not well equipped enough to discuss these matters with their children at home and, when these children meet their peers, they act based on misleading information. A person who knows that adolescence is a stage of curiosity and experimentation will understand why learners today are at such risk of drug or substance abuse as well as accidents.

\section{Methods AND Materials}

The research design that was used in this study was descriptive exploratory design that aimed at collecting information from participants on their ideas and opinions relating to sexual reproductive health. The qualitative method sought in-depth understanding of complex psychological issues and are most useful for answering humanistic "why?" and "how?" questions. The study was carried out through focus group discussions and interviews. A case study approach was utilised. The research techniques ensured representation of ideas, opinions, suggestions and recommendations concerning sexual reproductive health rights.

The sample size for this study was 52 parents of both sexes and those who have adolescents. In this study, the researcher used purposive sampling to obtain information from the parents with the understanding that parents are the ones who always tend to initiate the discussion with their adolescents on issues concerning reproductive health. The researcher selected the participants who best met the purpose of this study. Confidentiality was observed to both the information provided and to participants. Written consent was obtained from the participants to tape the interview. Participants were at any point free to withdraw from the study even though none did so. 


\section{ReSUlts}

\subsection{Sample Size, Age and Sex Distribution}

A total of 52 respondents took part in the study. The sample comprised of two focus group discussions (24) and twenty eight respondents who provided valuable information with questionnaires. All the respondents for this study were parents. $62 \%$ of the respondents were female and $38 \%$ were male. The respondents age respondents from 24 to 62 years. Their mean age was 43,88 with a standard deviation of 7,48 .

\subsection{Respondents Level of Education}

\begin{tabular}{|l|l|l|}
\hline Education & Frequency & Percent \\
\hline None & 6 & 11.5 \\
\hline Primary & 8 & 15.4 \\
\hline Secondary & 32 & 61.6 \\
\hline College and Above & 6 & 11.5 \\
\hline Total & $\mathbf{5 2}$ & $\mathbf{1 0 0}$ \\
\hline
\end{tabular}

\subsection{Respondents Knowle dge of Sexuality}

\begin{tabular}{|l|l|l|}
\hline Knowledge & Frequency & Percent \\
\hline Knowledgeable & 39 & 25 \\
\hline No Knowledge & 13 & 75 \\
\hline Total & $\mathbf{5 2}$ & $\mathbf{1 0 0}$ \\
\hline
\end{tabular}

\subsection{Parents}

Parents do not communicate sexual information effectively to their children. Mothers do attempt to communicate although they begin when the child is already in trouble with sexual desires but fathers are unsupportive. Parents are not formally involved by the schools with regard to the sex education programmes. Culture and religion hinder parents from talking to their children about sexuality matters. Parents would like to be primary sex educators of their children. Parents would like to be involved by the schools in sex education programmes.

\section{Social barriers}

\section{Gender differences}

Gender is one of the barriers to effective communication between parents and adolescents concerning reproductive health issues as it revealed in this study. Parents fail to communicate with their children of the opposite sex on issues based on sexuality like physical development, STIs, Puberty and condom use. This is a problem for all parents of both sexes as gender roles tend to be the barrier that tends to face parent-adolescent communication concerning issues of reproductive health. In most cases it has observed that this kind of communication tends to be gender based as parents prefer to speak or discuss with children of the same gender. Mothers prefer to talk with their daughters and fathers prefer to talk with their sons. If communication between parents and children of the opposite sex, then this communication take place in the context of:

\section{Shame}

Both men and women interviewed explained that it was shameful for them to discuss with their children of the opposite sex issues of STIs, condom use, HIV/AIDS and physical development. "I can't communicate with my child of any sex, about sexually transmitted Infections because it is shame for me. My child will not understand me as she or he will feel shame too. If she is a girl she might feel something different like I need to have an affair with her, and for the boy, he will not understand me". " ....it is difficult to tell my child that your voice has changed, I feel shy, but since I see physical changes, I started emphasizing on directing him to act responsibly as an adult like cleanliness". "I have never discussed with my children issues of puberty because it is shame for me as a parent to talk about those issues and traditionally it is abomination for a parent to talk with his children about these issues. These are the responsibilities of elders like grandfathers and grandmothers and not of a parent as they teach a child how to act and behave as an adult". 


\section{Fear that their children will feel they want to have an affair with them.}

Gender also is a barrier to parent-adolescent communication as other parents both male and female explained that it is difficult for them to communicate with their children on some issues of reproductive health as their children are of opposite sex. Parents feared that their children will feel that their parents want to have an affair with them especially when the discussion is about sexuality. "I can't communicate with my child of any sex, about Sexually Transmitted Infections because it is shame for me. My child will not understand me. She or he will feel shame too. If she is a girl she might feel that I want to have an affair with her, and for the boy, he will not understand me". "I couldn't talk with my child about physical development.

\section{Education}

The education levels of parents tend to be a barrier to parent- adolescents communication concerning reproductive health issues especially concerning sexuality topics. Parents with a high level of education, although they fail to communicate with their children orally or face to face, they decide to use other means of communication, like giving them learning materials to ensure that their children understand and get to know all the information about reproductive health issues compared to others with low education who have not seen other option to communicate with their children. "I have never tried to discuss directly with my child issues of STIs although I see feature of physical development. But I used to bring him materials like books and brochures that explain about those diseases, and this started when I saw that my child was changing physically. I know that my child understands because the materials are written in a language he knows. Everything about prevention and transmission, are explained in those books and brochures". (P10- Woman) "I have never tried to talk with my child about STIs because I know that he knows everything as there are many things to give information including mass Medias of which I think for my child knows everything. I did not have to tell him ways of preventing transmission because I thought in so doing I would get him to have sex".

Fear that they will direct their children to engage into sexual experimentation. Some parents explained that it is difficult to discuss with their children issues of reproductive health as they fear that they can direct them into engaging sexual activities. For parents to discuss with their children issues of puberty, condom use, STIs and HIV prevention and early pregnancy prevention is like they are directing their children to engage in or to practice sexual activities. "I can't talk with my child issues of puberty and physical development because it will be like I'm directing him or her to engage into sexual activities" (P1- Woman). "If I need to talk with my child on issues of how to prevent STIs, HIV and early pregnancy I will tell her to abstain only and not about other ways because if I tell her about other ways, it will be like I will be directing and encouraging her to engage into sex activities" (P2Woman). "It is very difficult for me as a parent to tell my child about consistent condom use, STIs and HIV prevention and early pregnancy prevention because when I teach him ways to prevent it is like I will be directing him to practice sex".

Feelings that their children are still young to know about reproductive health issues. Among the reasons why parents communicate less or not at all with their children on issues of reproductive health is that many parents have a feeling that the children are still too young. They think it is not yet a time for them to know about these things because they are too young. "My child is still too young to talk with him about STIs. These are the diseases of adults, hence it is shame to talk about them with her, especially about how they are transmitted and of ways to prevent them" (P2- Woman). "........You as a parent you should feel shame to talk with your child about STIs, Condom use, and pregnancy prevention methods because these children are still too young to know all of these."

\section{Fee lings that their children know the facts.}

Other parents interviewed explained that they don't discuss issues of HIV and AIDS, early pregnancy, condom use and STIs with their children because they think that they know everything due to advanced science and technology as there are televisions, internets and different radio stations that talk about those issues, then it is easy for their children to learn much from other sources. "Do you think that our children they don't know about how pregnancy, STIs and HIV/AIDS is obtained and how to prevent it? I know they know because of science and technology that is why I can't talk with them although I feel shame too." "I know my child knows everything concerning condom use, early pregnancy STIs, HIV and AIDS because of the nature of the living we are which is advanced in terms 
of science and technology where a child can get information from brochures, televisions, internets and journals, that is why I can't talk with child anymore." "...I know that even my child know ways of preventing pregnancies because the life that we had our children knows everything."

\section{Marital Status}

Being married tends to be a barrier to communication between parents and their adolescents concerning reproductive health issues as parents within the marriage tend to have different perception about who has the responsibility to communicate with the child concerning reproductive health issues like STIs and HIV/AIDS. Parents who are divorced assume that they have all the responsibilities in ensuring that their children, regardless their sex, have all the information about different issues of reproductive health. They do not communicate in a direct way with so as their children. They give them reading materials. "Mmhhh! I can't talk with my child of opposite sex issues of STIs, firstly I don't know even how to start, and even if I want to talk with him I will direct him to ask his father". "It will depend with the sex of the child, if the child is a girl then I will talk with her, and if it is a boy then his father has to talk with, because it is easy for each parent to talk with the child of his or her sex. It is shame for me to talk with a child of the opposite sex issue relating to reproductive organs". Parents who are divorced and remain as single parents, communication with their children regardless their sex tends to be more direct compared to those in marriage as they tend to have different way of ensuring that their children get the right information. "I prefer to use brochures and periodicals which explain how STIs are transmitted. I started soon after I saw my child changing physically. He was at the age of having sexual desires which would force him into sexual experimentation".

\section{Cultural Barriers}

\section{Traditional norms}

All the interviewed participants explained that traditional norms of their culture were not friendly for them to discuss issues of puberty with their children as it was not allowed because it is based on sexuality discussion. They believe that they are not the right people to talk with their children about those issues as their cultural norms allow this activity to be handled by senior or elders like grandfathers, grandmothers, aunts and uncles. Hence this is the barrier that makes them fail to talk with their children about issues of puberty. "My traditional norms do not allow me to direct my child on issues of puberty because it is shame for parent to talk about puberty to his or her child. This needs to be done by grandfathers and grandmothers" "I can't talk with my child of any sex issues about puberty because my traditional norms do not allow me to do so. This activity is done by other guardians". "No, I can't talk with my child of any sex issues of puberty because my traditional norms prohibit me as a father. I $t$ is an insult for me as a parent to discuss issues like these."

\section{Religion}

Religious belief that parents interviewed hold tend to be the barrier to communication between parents and their adolescents on issues of sexuality like STIs, HIV and early pregnancy prevention as well as condom use. They use statements that are provided in holy books (Bible and Quran) to guide their children to avoid sexual risk behaviors like multiple partners. Parents tend to use religious teaching rather than direct communication about condom use. Parents refuse totally to talk with their children about condom use. "My religion help me very much in communicating with my child as it prohibits adultery which is a sin to God. My religion also prohibits me to use condom, so I can't direct my child about condom use and I don't like him to use condom as it is not the will of God". "It is abomination to talk with my child of any gender issues of puberty even my religion prohibit me to talk those issues. This job is provided to seniors who are not the parents of the child".

\section{Occupation}

Occupation in which other parents are engaged to tend to be the barrier for parents to discuss with their children issues of reproductive health as they have little time to be with their children. "My job keeps me very busy. I used to travel frequently, which make me to have little time to be with my children and discuss with them. Furthermore my tradition prohibits me to talk with them about issues of reproductive health like puberty" (P6- Man). "My jobs are many and I travel frequently. Time is a limiting factor". "Truly economic activities are very tight, but when I get time I talk with my child about physical development." 


\section{Wealth (What parents own)}

The role of wealth in parent- adolescent communication was not found, as parents interviewed declared that it does not have any effect to hinder communication between them and their children. "What you own has nothing to do with preventing your child from risk sexual behaviors. What is needed is for parents to send a message to their children about risk sexual behaviors so that they know what to do." "Wealth is not necessary, what is required is for my child to know and understand risk sexual behaviors. Educating her doesn't cost anything."

\section{Income}

Income of parents doesn't have any effect to the parent- adolescent communication concerning reproductive health issues as parents interviewed explain that their income was nothing to do with the discussion with their children as communication doesn't need money. "Issues of HIV/AIDS are discussed regardless of income because this disease doesn't select people who have or who do not have. So I used to tell my children that AIDS KILLS." "Income is nothing because if I have or do not have it, still children need to be told and mother is the only person to tell them everything. Money is not needed." "Income is never needed to discuss with my child issues of HIV/AIDS; to me I think this discussion is free as income is not needed. What is mostly important is to use strategies that will help a child to understand the issues of HIV/AIDS."

\section{DisCUSSIONS}

Communication within the family appears to be particularly important during the adolescent years especially concerning sexual and reproductive health issues. Family communication affects adolescent identity formation and role-taking ability (Cooper et al., 1982). Cooper et al. suggest that adolescents who experience the support of their families may feel freer to explore identity issues.

The research findings indicate that some communication takes place on particular reproductive health issues but not others. In particular parents fail to communicate with their adolescent children on sensitive issues of sexuality like condom use, puberty, STIs, and physical development, but do so on less sensitive ones such as the effects of HIV. This implies that communication between parents is not comprehensive and informative when it comes to preparing their growing children to handle the emerging sexual needs they feel responsibly, so that they can minimize the risks for early pregnancy, STIs and HIV infection. Furthermore the study found that that even when communication takes place it is inadequate as the process does not always involve direct conversation between parents and their adolescents. Parents prefer using indirect communication methods like providing reading materials to their children. This implies that the children do not get direct assistance and clarification of complex information they come across as they read the material.

It can be concluded that the children do not benefit fully from such communication. For parents who try to have direct communicate with their children they tend to use threats rather than giving explanations and direction on "what to do and how." Hence the barriers revealed by the study tend to relate to communication about sexuality issues like STIs, puberty, physical development, early pregnancy and condom use than other reproductive health issues because discussion between 33 parents and their children of both sexes tend to be difficult on all issues based on sexuality.

\section{Cultural barriers}

It is worth noting that the role of traditional norms that limit communication between parents and their children on issues of sexuality was also found to operate in other societies, as a study done in Kenya (Mbugua 2007) reported. Parents are not expected to discuss with their children issues of physical development and puberty. This task is given to other people who are senior like grandmothers, grandfathers, aunts and uncles. Not only is found to be shameful by parents, society finds it to be an abomination for parents to talk about these issues with their children as the discussion will be about sexuality. In this era of the HIV epidemic societies need to reexamine their cultural norms and do away with those that are not helpful in confronting the new challenges, particularly because urbanization has meant that such senior elders may not be within easy reach. As for religious beliefs that prevent parents from discussing with their children about use of condoms, it is important religious organizations find more effective ways of teaching about prevention other than the injunction not to sin which is not heeded even by some religious leaders. At least schools should be 
allowed to deal with this subject of prevention of early pregnancy, STIs and HIV infection by teaching about scientifically proved methods.

\section{Social barriers}

This study found that when communication takes place it tends to be gender based as parents prefer to speak or discuss with children of the same gender as themselves. Mothers prefer to talk with daughters and fathers prefer to talk with their sons. This finding is similar to that of Eisenberg et al, (2006), Education is a factor in parent- adolescent communication. Parents with low levels of education tend to limit the communication process. Parents don't want their children to engage in early sexual experimentation or to do anything sexual. They avoid talking to their children about sex and to confront them about what they believe the children are doing concerning sexuality. Parents fear that any discussion of this nature can lead the children to engage into sexual experimentation. This finding is similar to that of another Kenyan study (Poulsen et al, 2010). This suggests parents need to be educated to understand that adolescents are not young as at that age, they are aware of sexual needs, and some of them may be sexually active. They need all the necessary education and information before it is too late to correct any mistakes. Parents with high level of education do not talk to their children as they assume that they know each and everything from television, radio, and the internet. So they let them find for themselves the information they want. While this is going on parents need to check with their children because not everything they come across in the social media is correct, and misinformation abounds. The division of roles and responsibility for guiding children among couples is different, whereby mothers feel that it is fathers who are supposed to tell their sons this, and vice versa. This is particularly the case in the context of pressing economic activities which mean that either the father or mother may not be available to sit down and discuss issues with an apparently perturbed daughter or son. Divorced parents in turn assume that they have all the responsibility in ensuring that their children regardless their sex have all the information about different issues of reproductive health and will use any methods of communication. These results are similar to that of South African study (Wang, 2000).

A growing body of research conducted with young people in developing country contexts has confirmed that parents can influence the sexual decision-making of their adolescent children ( KumiKyereme et al., 2001; Zhang et al., 2007; Blum and Mmari, 2005; Mohammadi et al., 2007; Ahmed and Blum, 2008). These findings are consistent with a considerably larger body of evidence from the US that has found that parent child connectedness and communication plays an important role in delaying sexual initiation, reducing multiple partner relations and increasing condom use (Miller et al., 1998; Meschke, Bartholomae and Zentall, 2000; DiClemente et al, 2001; Hollander, 2007; Lederman, Chan and Roberst-Gray, 2004; Ryan et al., 2007; Aspy et al., 2007; Kirby et al., 1991; Whitaker et al., 1999).

\section{CONClusion}

There is enough evidence from around the world that parents matter, and that supportive communication between parents and children enables young people to make a safe and confident transition to adulthood. This study, probed parental perspectives, suggests that parents are indeed concerned about their children's transition into sexual life, but are constrained by traditional norms, lack of information and limited skills from communicating with and providing them the supportive environment needed to make this transition. It is important that efforts are initiated that enable parents to overcome these constraints. It can therefore be noted that dialogue between parents and adolescents good reproductive health. This is more important in the current environment where adolescents are at greater risk of reproductive health problems. In a society like Zimbabwe where reliance on extended families especially in the urban areas is becoming less viable, parents remain the first and most important persons in socialisation of their children in all aspects of life including sexuality.

\section{RECOMMENDATIONS}

Parents should start discussing sexuality with their adolescent children early. This should be done much early before the adolescents are sexually active.

Educators of adolescents' reproductive health should educate parents on the need to discuss with their children the changes in sexual characteristics of adolescents. 
Parental Knowledge, Attitudes and Perceptions towards Adolescent Sexual Reproductive Health in Bulawayo

$>$ There is need to implement and test the effectiveness of interventions designed to improve parental- adolescents communication and interaction.

$>$ Parent- adolescent communication on sexuality should be promoted through various mechanisms. Among these is promoting school sex education homework assignments designed to be completed by both parents and adolescents to enhance parent-adolescent communication.

$>$ Mass media should continue to be used to solicit involvement of parents in providing sex education to their children and hence minimizing the cultural bottlenecks related to sexuality education.

Sensitization to both parents and adolescents needs to be done so that any of them can initiate the discussion about sexual and reproductive health issues.

$>$ Training and evaluation about sexuality to parents on how to communicate with their children of the opposite sex

\section{REFERENCES}

Abraham, L. and K.A. Kumar. (1999). "Sexual experiences and their correlates among college students in Mumbai city, India." International Family Planning Perspectives, 25(3): 139-46.

Alexander, M., L. Garda, S. Kanade et al. (2006). "Formation of partners hips among young women and men in Pune District, Maharashtra." New Delhi: Population Council.

Aspy C.B., S.K. Vesely, R.F. Oman et al. (2007). "Pare ntal communication and youth sexual behaviour." Journal of Adolescence, 30(3): 449-66.

Bettinger, J.A., D.D. Celentano, F.C. Curriero et al. (2004). "Does parental involvement predict new sexually transmitted diseases in female adolescents?" Pediatrics and Adolescent Medicine, 158: 666-670.

Biddlecom, A., K. Awusabo-Asare and A. Bankole. (2009). "Role of parents in adolescent sexual activity and contraceptive use in four African countries." International Perspectives on Sexual and Reproductive Health, 35(2): 72-81.

Blanc,A.K.,(2006) The changing context of sexual initiation in Sub-Saharan Africa. Population and development review.32(4):699-728.

Blum, R.W. and K.N. Mmari. (2005). "Risk and protective factors affecting adolescent reproductive in developing countries." Geneva: World Health Organization.

Cui, N., M. Li. and E. Gao. (2001). "View of Chinese pare nts on the provision of contraception to unmarrie d youth." Reproductive Health Matters, 9(17): 137-145.

Di Clemente R.J., G.M. Wingood, R. Crosby et al. (2001). "Parent-adolescent communication and sexual risk behaviours among African American adolescent fe males." Journal of Pediatrics, 139: 407-412.

Garda, L. and M. Alexander. (2009). "Parent-child connectedness: A pressing need to be addressed in rural and urban India." Paper presented at XXVI IUSSP International Population Conference, Marrakech, Morocco.

Kiragu, K., C. Watson, M. Muhwezi et al. (2007). "Straight Talk Campaign in Uganda: Parent Survey." Washington, DC: Population Council.

Kuate, D. B. Family environment and premarital intercourse in Bandjoun (West Cameroon). Arch Sex Behav 2012, 41:351-361.

Lambert, H. and K. Wood. (2005). "A comparative analys is of communication about sex, health and sexual health in India and South Africa: Implications for HIV prevention." Culture, Health and Sexuality, 7(6): 527-541.

Lederman, R.P., W. Chan and C. Roberst-Gray. (2004). "Sexual risk attitudes and intentions of youth aged 12-14 years: survey comparisons of parent-teen prevention and control groups." Behavioural Medicine, 29(4): 155-163.

Li, X., B. Stanton and S. Feigelman. (2000). "Impact of perceived parental monitoring on adolescent risk behaviour over 4 years." Journal of Adolescent Health, 27: 49-56.

Luwaga, L.C.N. (2004). "Parent-adoles cent communication on sexuality in the context of HIV/AIDS in Uganda: An exploratory study." Bergen: Research Centre for Health Promotion. 
Mahajan, P. and N. Sharma. (2005). "Parents attitude towards imparting sex education to their adole scent girls." Anthropologist, 7(3): 197-199.

Mehra, S., R. Savithri and L. Coutinho. (2002). "Sexual behaviour among unmarried adolescents in Delhi, India: Opportunities despite pare ntal controls." Paper presented at the 2002 IUSSP Regional Population Conference, Bangkok, June.

Meschke, L.L., S. Bartholomae and S.R. Zentall. (2000). "Adolescent sexuality and parentadolescent processes: Promoting healthy te en choices." Family Relations, 49: 143-154.

Mohammadi, M.R., S. Alikhani., F.K. Abadi Farahani et al. (2007). "Parents attitudes towards adolescent boy's reproductive health needs and practice in Tehran." Iran Journal of Psychiatry, 2: 13-24.

Owuor, T.(2003), Parental presence and adolescent reproductive health among the Nairobi urban poor. Journal of Adolescent Health 33(5):369-377.

Ryan, S., K. Franzetta, J. Manlove et al. (2007). “Adolescents' dis cuss ions about contrace ption or STDs with partners before first sex." Perspectives on Sexual and Reproductive Health, 39(3): 149-157.

Shekhar, M., S. Ghosh and P. Panda. (2007). "Exploring Safe Sex Awareness and Sexual Experiences of Adolescents in Patna." Economic and Political Weekly, 42(48): 48-55.

Soletti, A.B., V. Guilamo-Ramos, D. Burnette et al. (2009). "India-US collaboration to prevent adolescent HIV infection: The feasibility of a family-based HIV-pre vention intervention for rural Indian youth." Journal of the International AIDS Society, 19:12(1):35.

Vandenhoudl,H., Youth pre vention activities in Western Kenya: Families Matter Program, paper presented at the Interagency Youth working group meeting, Washington, D.C, December 6, 2003.

Vondrasek C.(2005). Parental factors and sexual risk taking among young people in Cote d'Ivoire. African Journal of Reproductive Health.

Whitaker, D.J. and K.S. Miller. (2000). "Parent-adolescent discussions about sex and condoms: Impact on peer influences of sexual risk behavior." Journal of Adolescent Research, 15(2): 251-273.

WHO (2007). Summaries of Projects in Developing Countries Assisting the Parents of Adolescents. Geneva,WHO.

World Health Organization (WHO). (2007). "Helping parents in developing countries improve adolescents' health." Geneva: World Health Organization.

Zhang, L., X. Li., I. Shah et al. (2007). "Parent-adolescent sex communication in China." European Journal of Contraception and Reproductive Health Care, 12(2): 138-147.

\section{AUTHORS' BIOGRAPHY}

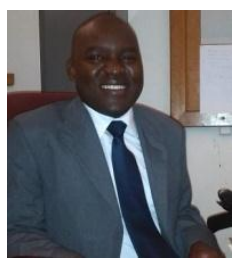

Mathew Svodziwa, is a Lecturer at Bulawayo Polytechnic in Zimbabwe.

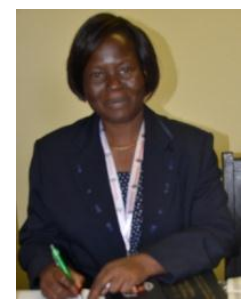

Faith Kurete, is a Lecturer at Bulawayo Polytechnic in Zimbabwe.

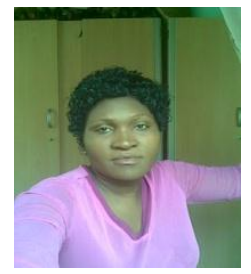

Loveness Ndlovu, is a Lecturer at Bulawayo Polytechnic in Zimbabwe. 